\title{
BMJ Open Does cannabis legalisation change healthcare utilisation? A population- based study using the healthcare cost and utilisation project in Colorado, USA
}

\author{
Francesca N Delling, ${ }^{1}$ Eric Vittinghoff, ${ }^{2}$ Thomas A Dewland, ${ }^{3}$ Mark J Pletcher, ${ }^{2}$ \\ Jeffrey E Olgin, ${ }^{1}$ Gregory Nah, ${ }^{1}$ Kirstin Aschbacher, ${ }^{1}$ Christina D Fang, ${ }^{1}$ \\ Emily S Lee, ${ }^{1}$ Shannon M Fan, ${ }^{1}$ Dhruv S Kazi, ${ }^{1}$ Gregory M Marcus ${ }^{1}$
}

To cite: Delling FN, Vittinghoff $\mathrm{E}$, Dewland TA, et al. Does cannabis legalisation change healthcare utilisation? A population-based study using the healthcare cost and utilisation project in Colorado, USA. BMJ Open 2019;9:e027432. doi:10.1136/ bmjopen-2018-027432

- Prepublication history and additional material for this paper are available online. To view please visit the journal(http://dx. doi.org/10.1136/bmjopen-2018027432).

Received 22 October 2018 Revised 23 March 2019 Accepted 17 April 2019

Check for updates

(c) Author(s) (or their employer(s)) 2019. Re-use permitted under CC BY-NC. No commercial re-use. See rights and permissions. Published by BMJ.

${ }^{1}$ Medicine (Cardiology), University of California, San Francisco, California, USA ${ }^{2}$ Epidemiology and Biostatistics, University of California, San Francisco, California, USA

${ }^{3}$ Medicine, Oregon Health \& Science University, Portland, Oregon, USA

Correspondence to Professor Gregory M Marcus; marcusg@medicine.ucsf.edu

\section{ABSTRACT}

Objective To assess the effect of cannabis legalisation on health effects and healthcare utilisation in Colorado (CO), the first state to legalise recreational cannabis, when compared with two control states, New York (NY) and Oklahoma (OK).

Design We used the 2010 to 2014 Healthcare Cost and Utilisation Project (HCUP) inpatient databases to compare changes in rates of healthcare utilisation and diagnoses in $\mathrm{CO}$ versus NY and $\mathrm{OK}$.

Setting Population-based, inpatient.

Participants HCUP state-wide data comprising over 28 million individuals and over 16 million hospitalisations across three states.

Main outcome measures We used International Classification of Diseases-Ninth Edition codes to assess changes in healthcare utilisation specific to various medical diagnoses potentially treated by or exacerbated by cannabis. Diagnoses were classified based on weight of evidence from the National Academy of Science (NAS). Negative binomial models were used to compare rates of admissions between states.

Results In CO compared with NY and OK, respectively, cannabis abuse hospitalisations increased (risk ratio (RR) $1.27,95 \% \mathrm{Cl} 1.26$ to 1.28 and $\mathrm{RR} 1.16,95 \% \mathrm{Cl} 1.15$ to 1.17 ; both $\mathrm{p}<0.0005)$ post-legalisation. In $\mathrm{C} 0$, there was a reduction in total admissions but only when compared with OK (RR $0.97,95 \% \mathrm{Cl} 0.96$ to $0.98, \mathrm{p}<0.0005$ ). Length of stay and costs did not change significantly in CO compared with NY or OK. Post-legalisation changes most consistent with NAS included an increase in motor vehicle accidents, alcohol abuse, overdose injury and a reduction in chronic pain admissions (all $p<0.05$ compared with each control state). Conclusions Recreational cannabis legalisation is associated with neutral effects on healthcare utilisation. In line with previous evidence, cannabis liberalisation is linked to an increase in motor vehicle accidents, alcohol abuse, overdose injuries and a decrease in chronic pain admissions. Such population-level effects may help guide future decisions regarding cannabis use, prescription and policy.

\section{INTRODUCTION}

Over 147 million people, or 2.5 per cent of the world's population, use cannabis
Strengths and limitations of this study

- The study focuses on the impact of recreational cannabis legalisation on hospital admissions instead of its legal or societal effects.

- This study utilises state-wide hospital admission data comprising over 28 million individuals and over 16 million hospitalisations.

- Effects on hospital admissions are evaluated in Colorado, the first state to legalise recreational cannabis, but compared with only two control states, New York and Oklahoma.

- Follow-up data after recreational cannabis legalisation only includes two subsequent years.

- Physician coding with International Classification of Diseases-Ninth Edition codes does not capture potentially important mediators related to cannabis use.

(marijuana), ${ }^{1}$ and more than 20 million Americans have reported the use of cannabis in the past 30 days. ${ }^{1}$ Because cannabis use is a federal crime, clinical studies have been challenging to pursue resulting in substantial knowledge gaps regarding actual health consequences. Thirty-three states and the District of Columbia now allow cannabis for the treatment of medical conditions. ${ }^{2}$ Of these, nine have recently legalised cannabis for recreational use. Given such pervasive policy changes, understanding potential shifts in healthcare utilisation is vital.

Recently, an extensive and rigorous summary of the current evidence on health effects of cannabis was developed by a committee of experts appointed by the US National Academy of Science (NAS) focusing on systematic reviews and high-quality primary research. ${ }^{3}$ The health endpoints assessed in the NAS summary included oncological, cardiometabolical, respiratory, 
immunological and psychiatric disorders as well as outcomes related to injury and death (ie, motor vehicle accidents). While the NAS summary represents a valuable starting place that makes use of the available data, we do not yet know whether that evidence, based largely on small studies, will translate into real-world ramifications after legalisation of recreational cannabis.

On 10 December, 2012, Colorado enacted Colorado Amendment 64, legalising recreational cannabis. Following this date, adults aged 21 or older could grow cannabis plants privately, legally possess all cannabis from these plants and give cannabis as a gift to other adults aged 21 or older. ${ }^{4}$ After 1 January, 2014, recreational cannabis could be legally purchased in retail stores. ${ }^{5}$ We hypothesised that changes in healthcare utilisation and diagnoses most consistent with NAS-based evidence occur when access to recreational cannabis becomes liberalised.

\section{METHODS}

We used the Agency for Healthcare Research and Quality (AHRQ)-funded Healthcare Cost and Utilisation Project (HCUP) database to measure inpatient healthcare utilisation and diagnoses in Colorado (CO) between 2010 and 2014. HCUP is a state-wide database containing all listed inpatient diagnoses and procedures, discharge status, patient demographics and charges for all patients, regardless of payer (eg, Medicare, Medicaid, private insurance, uninsured).

Comparisons were made with two control states in order to address possible secular trends. New York (NY), the most populous state with inpatient HCUP data available up to 2014 was selected, and, to counter this coastal and largely urban state, we also selected Oklahoma (OK), a predominately rural state directly adjacent to $\mathrm{CO}$ with HCUP hospitalisation data up to 2014. We used separate HCUP databases for CO, NY and OK. Unlike the National Inpatient Sample (NIS), which is (by definition) a sample, these state-specific databases include data from every actual admission, providing direct and complete information regarding all healthcare utilisation. Both primary and secondary diagnoses were extracted from each HCUP database for each admission used: up to 30 International Classification of Diseases-Ninth Edition (ICD-9) codes in $\mathrm{CO}, 25$ in $\mathrm{NY}$ and 16 in OK were provided for each encounter. Annual demographical data, including age, sex and race, was obtained from the US Census Bureau. Only month and year were available in all HCUP databases; as legalisation took effect on 10 December, 2012, hospitalisations following December 2012 were considered 'after' enactment of the law. Patients with missing information on age, sex, race and year of encounter were excluded.

Age, sex, race, income level and insurance payer were recorded at each healthcare encounter. Hispanic ethnicity was not coded in the OK State Department of Health data source. In order to use comparable categorisations across the three states, we separated race into white, black, Native American and other. In addition to the population-level variables age, sex and race, we obtained the following covariates at the individual level for each age, sex and race population strata: (1) income level, (2) proportion of HCUP individuals living in an urban versus rural setting, (3) with a diagnosis of tobacco use, (4) alcohol abuse and (5) psychiatric disorders. The latter three covariates were available from ICD-9 codes. Income level was categorised by quartiles using the median household income for each patient's postal code. Income level was not available for OK.

We first performed a validation analysis to assess changes in cannabis abuse diagnoses in $\mathrm{CO}$ after versus before recreational cannabis legalisation and compared those rates to changes in NY and OK over the same time period. To determine changes in overall healthcare utilisation, we assessed changes in total number of hospitalisations, length of inpatient stay and healthcare costs. We estimated the cost of each hospitalisation by multiplying the charges by a cost-to-charge ratio for the admitting hospital for the given year. For NY and CO, we used the all-payer cost-to-charge ratios provided by HCUP. When the all-payer cost-to-charge ratio was missing, we applied the average cost-to-charge ratio particular to state and year. As HCUP does not provide cost-to-charge ratios for $\mathrm{OK}$, we assumed a constant state-wide cost-to-charge ratio (0.3119 as derived from the Institute for Health and Socio-Economic Policy calculations of federal cost reports) to estimate healthcare costs in $\mathrm{OK}^{6}$; we then conducted a sensitivity analysis setting the cost-to-charge ratio for $\mathrm{OK}$ to equal to the average for $\mathrm{NY}$ and $\mathrm{CO}$ for the corresponding year. See online supplementary eMethods and eTable 1 for additional details.

We used ICD-9 codes (eTable 2) to assess changes in healthcare utilisation specific to various medical diagnoses potentially treated by or exacerbated by cannabis use as identified by the NAS summary. For each health endpoint of interest in the NAS review, the weight of evidence regarding the statistical association of recreational cannabis with a specific health endpoint or the therapeutic use of cannabis had previously been categorised into substantial, moderate, limited or no evidence. ${ }^{3}$ We opted to focus on NAS health endpoints with either substantial or moderate evidence.

Because diagnoses of alcohol or other substance abuse 'in remission' may have been incidental and not directly responsible for the hospitalisation, we performed a sensitivity analysis where we removed 'in remission' diagnoses from the group of ICD-9 codes defining alcohol abuse and other substance abuse (eTable 2).

Finally, to take into account the additional effect of recreational cannabis related to availability in retail stores we performed sensitivity analyses for all healthcare utilisation (total number of admissions, length of stay, costs) and NAS-evidence based outcomes investigations utilising 1 January, 2014, as the change of policy date. 
Certification to use de-identified HCUP data was obtained from the University of California, San Francisco Committee on Human Research.

\section{Statistical analysis}

Demographical and lifestyle characteristics are presented as mean $\pm \mathrm{SD}$ or $\mathrm{n}$ (\% of total admissions) and were compared between states using linear, logistical and multinomial models as appropriate. Rates of admissions for NAS diagnoses (with the population size as the denominator) were compared between states using negative binomial models.

To assess the effects of recreational cannabis legalisation, we used negative binomial models for the number of admissions, both overall and for particular diagnoses, with the log of the subgroup population sizes as an offset and the use of robust standard errors. Analogous linear models were used for length of stay and cost, which were both log-transformed in order to meet normality assumptions. All models were adjusted for age, race, sex, income level, urbanicity, diagnosis of alcohol abuse, tobacco use and psychiatric disorders. Adjustment for diagnosis of psychiatric disorders was not performed in the presence of a psychiatric outcome. In order to relax the linearity assumption, each 5 year age range was included as a separate category. To flexibly model secular patterns, year was included as a categorical rather than continuous variable (with a category for each year between 2010 and 2014). The crucial predictors in our model included an indicator for CO (versus the comparison state) and interactions of this indicator with two continuous linear spline basis functions, year-2012, and $\max (0$, year-2012). The main effect for $\mathrm{CO}$ estimated the between-state risk ratio (RR) in 2012, the first interaction estimated the slope of the between-state RR in 2010 to 2012, and the second estimated the change in slope after 2012. The first interaction was used to project the counterfactual 2013 to 2014 rates in $\mathrm{CO}$ that would be expected in the absence of legalisation, while the second was used to capture the legalisation effect. Like a standard interrupted time series (ITS) model, our model assumed that the between-state RR changed linearly at different rates before and after the intervention, but used the categorical indicator for year to relax the standard ITS assumption of piecewise linear trends in the underlying state-specific rates, substantially improving model fit. The same model was used in the sensitivity analysis using 1 January, 2014, (date of legal retail cannabis sales) as change of policy date.

A two-tailed $\mathrm{p}<0.05$ was considered statistically significant for the validation analysis (cannabis use) and analyses related to overall healthcare utilisation. In order to minimise false positive results and to account for secular trends that might differ across different populations, we assessed for validation in comparisons versus NY and then OK separately.

In order to address possible false positives due to multiple hypotheses testing in assessing the diagnoses with moderate or substantial evidence described in the
NAS document, we employed stringent standards: to be considered 'positive,' the comparisons between $\mathrm{CO}$ and each of the other two states needed to be in the direction expected from the NAS report, each exhibiting statistical significance using a two-tailed alpha of 0.05. A 'positive' designation also required absence of a statistically significant difference between NY versus OK using a two-tailed alpha of 0.05. All analyses were performed using Stata V.15 (StataCorp, College Station, Texas).

\section{Patient and public involvement}

The patients and public were not involved in the study.

\section{RESULTS}

\section{Baseline characteristics}

The total number of admissions between 2010 and 2014 are shown for each state in table 1. Patients in CO were generally younger, more commonly female, and less ethnically diverse. Wealth in $\mathrm{CO}$ was less equally distributed, with a higher proportion of individuals with lower income in CO compared with NY. Finally, admission rates were lower in $\mathrm{CO}$ than the control states for most NAS diagnoses, with exceptions for pre-diabetes and post-traumatic stress disorder.

\section{Study validation}

Over 2010 to 2014, the change in rates of cannabis abuse admissions after versus before recreational cannabis legalisation in 2012 was greater in $\mathrm{CO}$ than in NY and OK (RR 1.27, 95\% CI 1.26 to 1.28 and RR $1.16,95 \%$ CI 1.15 to 1.17 ; both $\mathrm{p}<0.0005$, respectively) (figure $1 \mathrm{~A}, \mathrm{~B}$ ). No significant changes comparing the two control states, $\mathrm{NY}$ and $\mathrm{OK}$, were observed over the same time period (RR $0.93,95 \%$ CI 0.87 to $1.00, \mathrm{p}=0.05)$.

\section{Inpatient healthcare utilisation after cannabis legalisation Number of admissions}

In unadjusted analyses, $\mathrm{CO}, \mathrm{NY}$ and $\mathrm{OK}$ had a similar number of total admissions before versus after recreational cannabis legalisation (figure 2A). After adjusting for covariates, there was a reduction of number of admissions following cannabis legalisation in $\mathrm{CO}$ when compared with OK (RR $0.97,95 \%$ CI 0.96 to $0.98, \mathrm{p}<0.0005$ ). The point estimate was similar when comparing $\mathrm{CO}$ to $\mathrm{NY}$, but did not reach statistical significance (RR 0.99, 95\% CI 0.98 to $1.01, \mathrm{p}=0.47)$. Results were similar in sensitivity analysis using 1 January, 2014, as change of policy date (CO vs OK: RR $0.96,95 \%$ CI 0.96 to $0.96, p<0.0005$ and CO vs NY: RR 0.99, $95 \%$ CI 0.96 to $1.02, \mathrm{p}=0.59$ ).

\section{Length of stay}

The median length of stay also remained similar after cannabis legalisation across the three states in unadjusted analyses (figure 2B). After adjusting for covariates, length of inpatient stay did not change significantly in $\mathrm{CO}$ following the law change when compared with each of the two control states $(1.75 \%$ annual reduction, $95 \% \mathrm{CI}$ $-12.25 \%$ to $10.01 \%$ in $\mathrm{CO}$ vs $\mathrm{NY}$, and $3.46 \%$ annual 
Table 1 Clinical characteristics of the three study states over the 2010 to 2014 period

\begin{tabular}{lllll}
\hline & Colorado & New York & Oklahoma & P value \\
\hline Total population & 5197237 & 19594599 & 3819383 & \\
\hline Total admissions & 2088909 & 11726283 & 2334988 & \\
\hline $\begin{array}{l}\text { Total admissions/1,000 population } \\
\text { Demographics }\end{array}$ & $402 \pm 0.27$ & $598 \pm 0.17$ & $611 \pm 0.40$ & 0.0001 \\
\hline Age - yr & $45 \pm 28$ & $49 \pm 28$ & $48 \pm 28$ & 0.0001 \\
\hline Male- no. (\%) & $870573(42)$ & $5087181(41)$ & $954848(43)$ & 0.0001 \\
\hline Race - no. (\%) & & & & 0.0001 \\
\hline White & $1548099(74)$ & $6406582(55)$ & $1879727(81)$ & \\
\hline Black & $102444(5)$ & $2083366(18)$ & $202420(9)$ & \\
$\quad$ Native American & $13143(1)$ & $36216(0.3)$ & $131714(6)$ & \\
\hline Other & $425223(20)$ & $3200119(27)$ & $121127(5)$
\end{tabular}

Lifestyle

Median household income, US\$ (quartiles for patient

0.0001

postal code) - no. (\%)

Quartile 1

$\begin{array}{llll}617537(30) & 3087192(28) & \text { No data } & \\ 558760(27) & 2623087(24) & & \\ 470051(23) & 2673153(24) & & \\ 251566(12) & 1717940(10) & 235685(14) & 0.0001 \\ 112715(5) & 652608(6) & 71931(3) & 0.0001 \\ 405(0.02) & 2487(0.02) & 480(0.02) & 0.0001\end{array}$

Quartile 2

Quartile 3

Tobacco use - no. (\%)

Alcohol abuse - no. (\%)

$2487(0.02)$

(\%)

Acute myocardial infarction - no. (\%)

\begin{tabular}{|c|c|c|c|}
\hline 39513 (1.9) & 271285 (2.3) & $60413(2.6)$ & 0.0001 \\
\hline $10963(0.5)$ & $58278(0.5)$ & $10245(0.4)$ & 0.0001 \\
\hline 27986 (1.3) & $194478(1.7)$ & $45602(2)$ & 0.0001 \\
\hline $294821(14)$ & 2301835 (20) & $465353(20)$ & 0.0001 \\
\hline 42544 (2) & $73407(0.6)$ & 24246 (1) & 0.0001 \\
\hline 65265 (3) & $429230(4)$ & $131343(6)$ & 0.0001 \\
\hline $14941(0.7)$ & $59686(0.5)$ & $13692(0.6)$ & 0.0001 \\
\hline $10933(0.5)$ & $40226(0.3)$ & $12147(0.5)$ & 0.0001 \\
\hline $158244(7.6)$ & 974856 (8.3) & 109305 (4.7) & 0.0001 \\
\hline $131426(6.4)$ & 595478 (5.2) & $139627(6.1)$ & 0.0001 \\
\hline 51913 (2.5) & $283280(2.4)$ & $65279(2.8)$ & 0.0016 \\
\hline 52348 (2.5) & 287735 (2.5) & $61411(2.6)$ & 0.0001 \\
\hline $213851(10)$ & $827932(7)$ & $190083(8)$ & 0.0001 \\
\hline 53652 (2.6) & 352533 (3) & $68385(2.9)$ & 0.0001 \\
\hline $2653(0.1)$ & $9482(0.1)$ & $3531(0.2)$ & 0.0001 \\
\hline $28582(1.4)$ & 75608 (0.6) & $17101(0.7)$ & 0.0001 \\
\hline $295(0.01)$ & $1090(0.01)$ & $135(0.01)$ & 0.0295 \\
\hline $4957(0.2)$ & $15646(0.1)$ & $4407(0.2)$ & 0.0001 \\
\hline $2842(0.1)$ & $7462(0.1)$ & $1742(0.1)$ & 0.0002 \\
\hline 39343 (1.9) & $144353(1.2)$ & $40938(1.8)$ & 0.0001 \\
\hline $3134(0.2)$ & $76789(0.7)$ & $730(0.03)$ & 0.0001 \\
\hline 63806 (3) & $16211(1.4)$ & $58706(2.5)$ & 0.0001 \\
\hline $140209(6)$ & 234160 (2) & 123563 (5) & 0.0001 \\
\hline
\end{tabular}

Continued

Brain haemorrhage - no. (\%)

Ischaemic stroke - no. (\%)

Metabolic syndrome \& diabetes - no. (\%)

Pre-diabetes - no. (\%)

Bronchitis - no. (\%)

Motor vehicle accidents - no. (\%)

Overdose injury - no. (\%)

Substance use disorder - no. (\%)

Anxiety disorders - no. (\%)

Anxiety disorders (except social anxiety) - no. (\%)

Bipolar disorders - no. (\%)

Depressive disorders - no. (\%)

Schizophrenia or other psychoses - no. (\%)

Positive symptoms of schizophrenia (ie, hallucinations) - no. (\%)

Post-traumatic stress disorder - no. (\%)

Social anxiety disorder - no. (\%)

Suicide - no. (\%)

Low offspring birth weight - no. (\%)

Maternal pregnancy complications - no. (\%)

Unemployment/low income - no. (\%)

Nausea or vomiting - no. (\%)

Chronic pain - no. (\%) 


\begin{tabular}{lllll}
\hline Table 1 Continued & & & \\
\hline & Colorado & New York & Oklahoma & P value \\
\hline Spasticity - no. (\%) & $5759(0.3)$ & $12517(0.1)$ & $5371(0.2)$ & 0.0001 \\
Short-term sleep - no. (\%) & $652(0.03)$ & $3364(0.03)$ & $357(0.02)$ & 0.0001 \\
Tourette syndrome - no. (\%) & $428(0.02)$ & $2784(0.02)$ & $336(0.01)$ & 0.0001 \\
Increased appetite \& weight gain - no. (\%) & $1022(0.05)$ & $3017(0.03)$ & $651(0.03)$ & 0.0001 \\
\hline
\end{tabular}

Admission diagnoses listed were based on those found influenced by cannabis in the 2017 NAS review. ${ }^{3}$ Substance use disorder includes dependence or abuse of alcohol, tobacco or other illicit drug. All variables pertain to the 2010 to 2014 period. Plus-minus values are means \pm SD. No. (\%) indicates number of admissions with a specific demographical/lifestyle characteristic or NAS diagnosis (\% of total admissions). A $P$ value less than 0.05 is considered statistically significant. NAS, National Academy of Science; yr, years.

reduction, $95 \%$ CI $-16.48 \%$ to $37.90 \%$ in $\mathrm{CO}$ vs $\mathrm{OK}$; $\mathrm{p}=0.30$ and $\mathrm{p}=0.20$, respectively). Length of stay did not change significantly in $\mathrm{CO}$ compared with the control states when we used 1 January, 2014, as change of policy date.

\section{Healthcare costs}

In unadjusted analyses, the three states exhibited similar total costs across the study time period (figure $2 \mathrm{C}$ and eTable 2). In multivariate analyses, healthcare costs remained similar in state comparisons $(2.99 \%, 95 \% \mathrm{CI}$ $-7.55 \%$ to $14.74 \%, \mathrm{p}=0.18 \mathrm{vs} \mathrm{NY}$ and $3.45 \%, 95 \% \mathrm{CI}$ $-7.31 \%$ to $15.46 \%, \mathrm{p}=0.16 \mathrm{vs} \mathrm{OK}$ ) after cannabis legalisation. No meaningful differences of healthcare costs were observed in the sensitivity analyses with 1 January, 2014, as change of policy date or when we assumed the cost-tocharge ratio for OK was equal to the average of the other states.

\section{Changes in specific diagnoses highlighted by the national academy of sciences}

Among the diagnoses with either substantial or moderate evidence of influence by cannabis per the NAS, we distinguished between: (1) a group with no statistically significant post-legalisation differences between control states (NY vs OK $\mathrm{p}>0.05$ ), suggesting that secular trends unlikely explained differences between $\mathrm{CO}$ and control states (figure 3) and (2) a group with significant differences in diagnoses post-legalisation between control states (NY vs OK $\mathrm{p}<0.05$ ) (eFigure 1).

In the $\mathrm{NY}$ versus $\mathrm{OK} \mathrm{p}>0.05$ group (figure 3 ), changes in rates of diagnoses after cannabis legalisation reflected NAS-based evidence for most health outcomes. Among the diagnoses most consistent with NAS-based evidence, there was an increase in hospital admissions for motor vehicle accidents, alcohol abuse, overdose injury and a

\section{Cannabis Abuse Admissions}

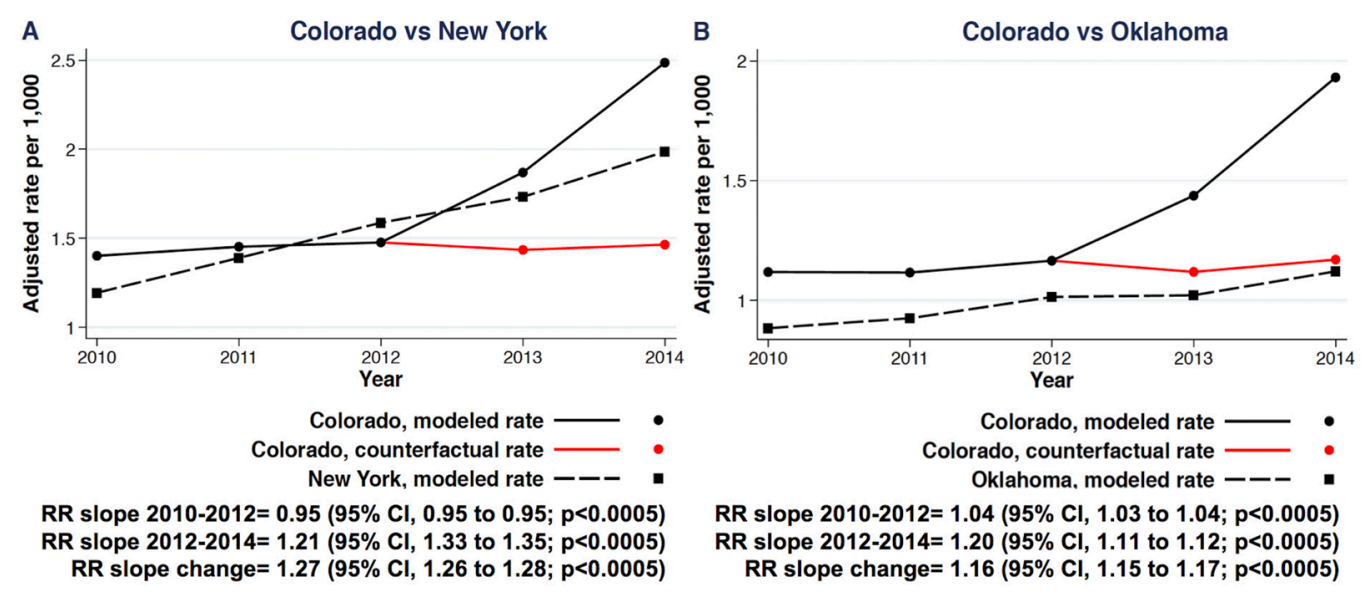

Figure 1 Multivariate adjusted rates of admissions for cannabis abuse over 2010 to 2014 in Colorado, site of implementation of the 2012 recreational cannabis legalisation policy, compared with New York (A) and Oklahoma (B), control states without cannabis legalisation. Red lines show the predicted rates of admissions for cannabis abuse had the cannabis legalisation policy not been instituted. The comparison between Colorado and the control states with regards to change of RR slope within the 2010 to 2012 period (pre-legalisation) and 2012 to 2014 period (post-legalisation), as well of the overall RR slope change, are also represented for the Colorado versus New York (A) and Colorado versus Oklahoma (B) comparisons. Red lines show the predicted rates of admissions for cannabis abuse had the cannabis legalisation policy not been instituted. Each model was adjusted for age, gender and race and state-level characteristics (see methods section for further details). RR, risk ratio; vs, versus. 


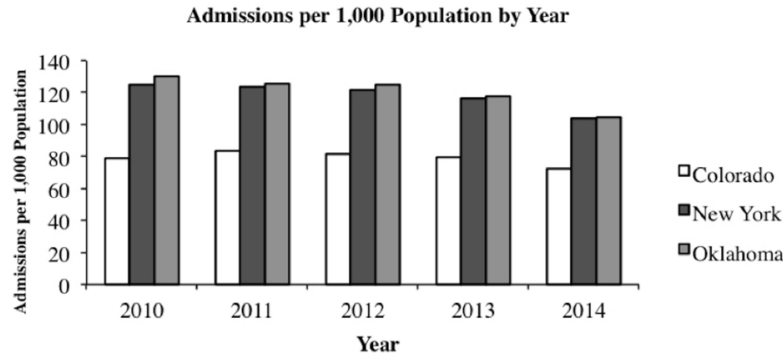

B
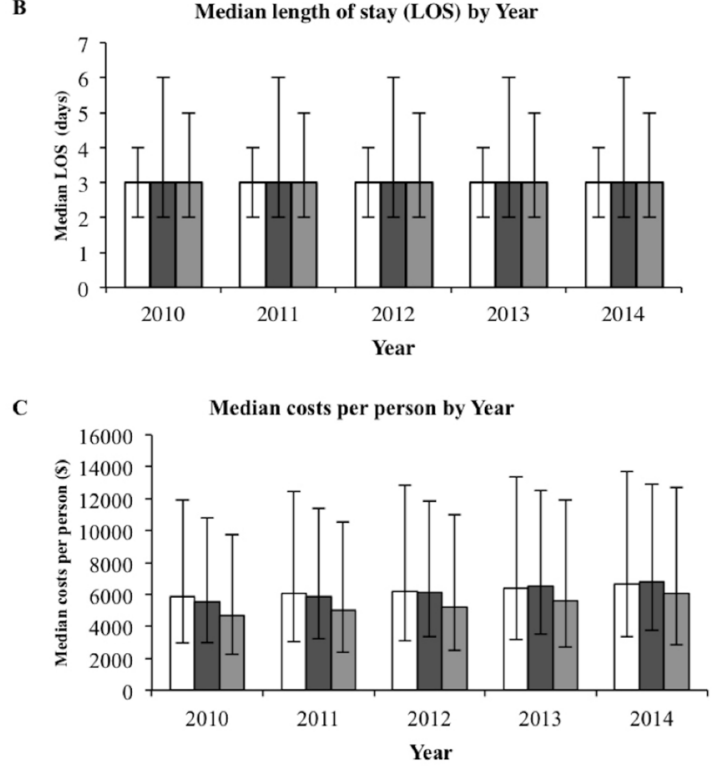

Figure 2 Healthcare utilisation over 2010 to 2014 in Colorado, site of implementation of the 2012 recreational cannabis legalisation policy, and in New York and Oklahoma, control states without cannabis legalisation. The total number of admissions/1000 population per year, the median length of stay per year and the median costs per individual per year are shown in panels A, B and C, respectively, for the three states. Error bars denote IQRs. LOS=length of stay.

decrease of chronic pain hospitalisations after recreational cannabis legalisation (each meeting statistical significance for both comparisons with control states). When using less stringent criteria $(p<0.05$ for both comparisons with $\mathrm{NY}$ and $\mathrm{OK}$, but where the relative risk for only one comparison was in the same direction as NAS), admissions for abuse of other substances and social anxiety disorder also increased with recreational cannabis legalisation (figure 3). Effects of cannabis liberalisation on psychiatric outcomes (schizophrenia and bipolar disorders) were either not consistent or weakly consistent with NAS (figure 3). When differences between the two control states (NY and $\mathrm{OK}$ ) were also significantly different (suggesting effects potentially related to secular trends) (eFigure 1), changes in diagnoses were mostly not concordant with NAS findings or one of the comparisons between $\mathrm{CO}$ and either NY or OK did not exhibit statistical significance. Changes of the risk over time with counterfactuals are shown for each NAS diagnosis in online supplementary eFigures 2-15.
In the sensitivity analysis using 1 January, 2014, as change of policy date (eTable 3 ), the main findings highlighted in the primary analysis using our stringent criteria (increase of admissions for alcohol abuse, motor vehicle accidents, overdose injury and decrease of chronic pain hospitalisations) again met the same criteria in favour of significant associations, except for overdose injury and chronic pain admissions (the latter meeting two of the three criteria described in the Methods). In sensitivity analyses removing 'in remission' diagnoses, no meaningful changes were observed (relevant to alcohol abuse and abuse of other substances).

\section{DISCUSSION \\ Principal findings}

Legalisation of recreational cannabis was associated with more cannabis abuse and minimal effects on overall healthcare utilisation. Changes in specific medical diagnoses post-legalisation reflected previously published substantial or moderate evidence on the health effects of cannabis, including an increase in motor vehicle accidents, alcohol abuse, overdose injury and a decrease of chronic pain admissions.

The increased frequency of hospitalisations for cannabis abuse in $\mathrm{CO}$ helps to validate the concept that legalisation would result in greater use.

Effects of recreational cannabis legalisation on healthcare utilisation appeared to be overall neutral. There was no evidence that either the length of stay or healthcare costs changed following liberalisation of recreational cannabis. There was a reduction of overall hospitalisations in $\mathrm{CO}$ when compared with $\mathrm{OK}$, but not compared with NY, potentially because unaccounted state-level characteristics may have driven overall admissions differently. Moreover, in the US healthcare system, hospitalised individuals tend to be sicker, with more severe forms of specific illnesses and higher inpatient costs compared with outpatients. Therefore, our conclusions on overall neutral effects of recreational cannabis on healthcare costs only reflect higher inpatient costs and not overall costs. Outpatient costs may include use of specific medications or substance detoxification programmes that are not captured in our study and may be more cost-effective compared with inpatient treatments.

Following legalisation of recreational cannabis, changes in rates of medical diagnoses reflected NAS-based evidence for most health outcomes. The absence of statistically significant differences in these outcomes between the two control states ( $\mathrm{NY}$ and $\mathrm{OK}$ ) over the same time period provides some evidence that these observed differences were less likely related to broader secular trends. After legalisation of recreational cannabis, there was an increase in motor vehicle accidents, alcohol abuse, overdose injury and a decrease of chronic pain admissions. In addition to information provided in the NAS summary, ${ }^{3}$ the association of cannabis with motor vehicle accidents has been highlighted by recent literature. ${ }^{78}$ Consistent 


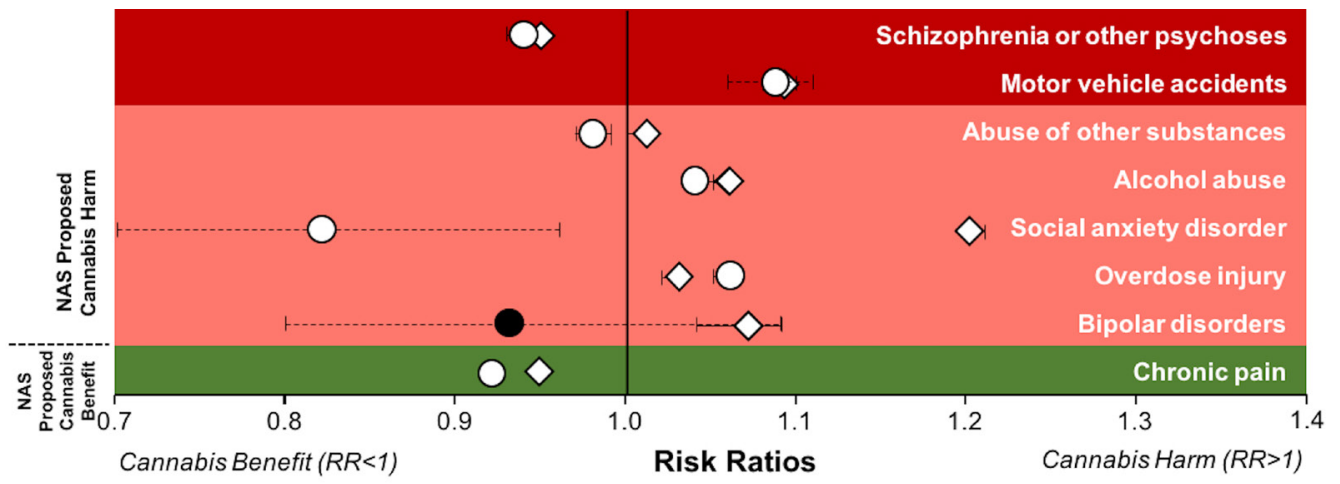

Figure 3 Health outcomes with substantial or moderate evidence of a statistical association with cannabis use based on the NAS summary statement. Medical diagnoses are identified based on a non-significant $(p>0.05)$ comparison between NY and OK (control states without cannabis legalisation) with regards to the change of admission rates for such diagnoses following cannabis legalisation. $P$ values for the comparison between $\mathrm{CO}$ (site of cannabis legalisation) and NY (rhomboid symbol), or between $\mathrm{CO}$ and $\mathrm{OK}$ (circular symbol) are shown as either $<0.05$ or $>0.05$ using a grey scale for evidence of cannabis benefit $\mathrm{RR}<1$ shown on the left) and cannabis harm (RR $>1$ shown on the right); $95 \% \mathrm{Cl}$ are shown for the comparison between $\mathrm{CO}$ and NY and between $\mathrm{CO}$ and $\mathrm{OK}$ as solid and dotted lines, respectively. Progressively darker shades of green or red indicate greater weight of evidence in the direction of proposed benefit versus harm, respectively, by the NAS. CO, Colorado; NAS, National Academy of Science; NY, New York; OK, Oklahoma; RR, risk ratio.

with our findings, a substantial bidirectional comorbidity between cannabis use and alcohol use has been previously demonstrated, resulting in a moderate level of evidence designation for this relationship per the NAS. ${ }^{9}$ Also compatible with the NAS summary, we report that recreational cannabis legalisation was associated with an increasing number of admissions for overdose injury. These included overdose of analgesics, barbiturates, sedative, hypnotics and psychotropic drugs (eTable 2). This finding underlines the association of cannabis use with other behaviours of drug addiction as highlighted by a recent cross-sectional survey of over 30000 community-living US adults. ${ }^{10}$ Finally, reduction of chronic pain, especially neuropathic pain, in cannabis users is well known, although prior literature has focused on medical rather than recreational cannabis. ${ }^{11} 12$

Except for social anxiety, the effects of recreational cannabis legalisation on admissions for psychiatric disorders (schizophrenia, bipolar disorder, depression) were weakly or not consistent with prior NAS-evidence. This finding may be related to residual confounding by statelevel characteristics, unaccounted secular trends or insufficient longitudinal follow-up.

We demonstrate that admissions for abuse of alcohol or other substances remains higher after recreational cannabis legalisation in a sensitivity analysis without 'in remission' diagnoses. Overall, these findings suggest that when the substance use disorder is in remission, it does not play a significant role in overall results.

In our primary analysis, we used 10 December, 2012, as the date of recreational cannabis legalisation. Following this date, private possession and growth of cannabis became legal in Colorado. After 1 January, 2014, recreational cannabis could be legally purchased in retail stores. ${ }^{5}$ To take the additional effect of retail sales of recreational cannabis into account, we performed sensitivity analyses using January 2014 as the 'change of policy' date. We did not observe any meaningful difference in the majority of the outcomes studied, suggesting that the effect of recreational cannabis on healthcare utilisation was independent of availability in stores.

\section{Strengths and weaknesses}

Although several studies have investigated the health effects of cannabis, ${ }^{13}$ they have relied on small sample sizes or have generated conflicting data. ${ }^{14-16}$ In contrast, our findings are derived from HCUP state-wide data comprising over 28 million individuals and over 16 million hospitalisations across CO, site of the 2012 Amendment 64, and two controls states without cannabis legalisation over the same time period. As NY and OK are very different geographically and demographically, we believe their inclusion as control states represents a strength of our manuscript. Specifically, their selection allowed us to demonstrate that the effects of recreational cannabis legalisation on healthcare utilisation were likely independent of living in an urban or rural setting.

Several limitations should be acknowledged. The HCUP database relies on physician coding; however, such coding for several medical diagnoses listed in table 1 have been shown to be highly specific with variable sensitivity ${ }^{17-20}$ and HCUP has proven to be a powerful 
tool in large population studies. ${ }^{20-24}$ Some potentially important mediators are not captured by ICD-9 codes, such as quantity of cannabis used or formulation (oral vs other), although these may be more relevant to identifying mechanisms and their absence would likely not lead to false positive results. Patients may be more likely to disclose cannabis use following legalisation of recreational cannabis and clinicians may be more inclined to test for it. Hence, we cannot completely exclude detection bias in the validation analysis showing an increase in cannabis abuse admissions following legalisation of recreational cannabis. Moreover, we cannot exclude that the increased frequency of cannabis abuse hospitalisations is simply related to severity of cannabis abuse rather than to a greater prevalence of cannabis use.

As with any observational study, we cannot exclude residual confounding as an explanation of our findings. However, we adjusted for conventionally recognised confounders as appropriate and as available, and, as this was largely a study of the same populations preand post-legalisation, many of the limitations inherent to conventional individual-level analyses are likely less relevant.

We did not select all US states as controls. Most states do not have HCUP data up until the end of 2014, each requires a separate HCUP application, and each carries significant costs. We acknowledge this represents a limitation as we could not fully account for secular trends that might influence results. However, we have adjusted all analyses for urbanicity and other state-level differences in order to minimise the impact of secular trends. We also acknowledge that $\mathrm{CO}$ had legalised medical cannabis prior to legalisation of recreational cannabis on 10 December, 2012. However, control states NY and OK had not. We could have selected control states that had already medicalised cannabis instead of NY and OK, but we acknowledge that complex differences exist among medical legalisation regimens among states. ${ }^{25}$ Hence, we believe selection of control states with diverse medical legalisation regimens would have primarily reduced our power to detect a real difference rather than contribute to any false positive spurious results. In our study, we demonstrate that legalisation of recreational cannabis is associated with more admissions for cannabis abuse or cannabis use disorder. Prior large, multi-state, population-based analyses have shown that legalisation of medical cannabis is associated with increased cannabis use, but not increased cannabis use disorder. ${ }^{26}$ One could speculate that medical cannabis consumption is more controlled and rationed when compared with recreational cannabis, the latter available in both retail stores and private homes and therefore perhaps more easily abused. Further studies are needed to quantify the amount of cannabis consumption (in grams) in states where only medical cannabis is legal versus states where both medical and recreational cannabis are allowed.

The HCUP database does not capture information regarding outpatient activity and therefore these findings are restricted to hospital-based medicine. However, even inclusion of outpatient data would not capture individuals with a specific condition or diagnosis who are not in formal treatment. Indeed, treatment databases do not capture all individuals in the population. This gap may be especially large for substance use disorders. Many individuals with substance use disorders may never enter treatment; even those who do more likely receive outpatient treatment, rather than a hospitalisation, so they may not have been included in our study.

Our use of stringent criteria in assessing changes related to NAS diagnoses may have been too conservative, leading to a sacrifice of false negatives in order to avoid false positives.

Finally, our study was limited to 2 year follow-up after recreational cannabis legalisation, and we therefore cannot comment on long-term effects that may result.

\section{CONCLUSIONS}

In conclusion, recreational cannabis legalisation is associated with an expected increase in cannabis use disorder. Overall effects on inpatient healthcare utilisation appear to be neutral, with subtle changes in various components of that utilisation likely occurring due to changes in the types of admissions observed. Measurable effects of recreational cannabis legalisation on increasing hospitalisations for motor vehicle accidents, alcohol abuse, overdose injury and reducing chronic pain admissions are consistent with previously published evidence. These data provide the first description of population-level effects that may help guide future decisions regarding cannabis policy, individuals considering using cannabis and physicians caring for those who may choose to consume it.

Contributors FND and GMM had full access to all of the data in the study and take responsibility for the integrity of the data and the accuracy of the data analysis. FND and GMM were responsible for the study concept and design. All authors (FND, EV, TAD, MJP, JEO, GN, KA, CDF, ESL, SMF, DSK and GMM) contributed to data acquisition, analysis and interpretation. EV, GN and GMM completed the statistical analysis. FND and GMM drafted the manuscript and were responsible for the critical revision of the manuscript for important intellectual content. GMM obtained funding for the study. All authors contributed to the administrative, technical and material support for the study and approved the final version of the manuscript.

Funding This work was supported by the Agency for Healthcare Research and Quality (AHRQ) and Health Care Information ( $\mathrm{HCl}$ ) Division of the Oklahoma State Department of Health.

Competing interests None declared.

Patient consent for publication Not required.

Ethics approval Ethical approval to use de-identified HCUP data was obtained from the University of California, San Francisco Committee on Human Research.

Provenance and peer review Not commissioned; externally peer reviewed.

Data sharing statement Data consist of de-identified participant data (ie, ICD-9 admission diagnoses) available from the Healthcare Cost and Utilisation Project (HCUP) for a fee. Information about how to obtain the data and for what time period can be found at: https://www.hcup-us.ahrq.gov/

Open access This is an open access article distributed in accordance with the Creative Commons Attribution Non Commercial (CC BY-NC 4.0) license, which permits others to distribute, remix, adapt, build upon this work non-commercially, and license their derivative works on different terms, provided the original work is 
properly cited, appropriate credit is given, any changes made indicated, and the use is non-commercial. See: http://creativecommons.org/licenses/by-nc/4.0/.

\section{REFERENCES}

1. Substance Abuse and Mental Health Services Administration. Behavioral health trends in the United States: Results from the 2014 National Survey on Drug Use and Health. Rockville, MD: U.S. Dept. of Health and Human Services, 2016. HHS Publication No. SMA 154927, NSDUH Series H-50.

2. National Conference of State Legislatures. State Medical Marijuana Laws 2016. 2018 http://www.ncsl.org/research/health/state-medicalmarijuana-laws.aspx (Accessed 14 Mar 2019).

3. Committee on the Health Effects of Marijuana, Board on Population Health and Public Health Practice, Health and Medicine Division, National Academies of Sciences, Engineering, and Medicine. The Health Effects of Cannabis and Cannabinoids: The Current State of Evidence and Recommendations for Research. Washington, DC: The National Academies Press, 2017.

4. Colo. Colorado Marijuana Legalization Initiative, Amendment 64, 2012.

5. Wark J. The New York Times. Up Early and in Line for a Marijuana Milestone in Colorado. 2014. https://www.nytimes.com/2014/01/02/ us/colorado-stores-throw-open-their-doors-to-pot-buyers.html

6. Institute for Health \& Socioeconomic Policy. Average Charge-to-cost Ratio by State. Silver Spring, MD: National Nurses United, 2012.

7. Rogeberg O. A meta-analysis of the crash risk of cannabis-positive drivers in culpability studies-Avoiding interpretational bias. Accid Anal Prev 2019;123:69-78.

8. Hostiuc S, Moldoveanu A, Negoi I, et al. The association of unfavorable traffic events and cannabis usage: a meta-analysis. Front Pharmacol 2018;9:99.

9. Blanco C, Hasin DS, Wall MM, et al. Cannabis Use and Risk of Psychiatric Disorders: Prospective Evidence From a US National Longitudinal Study. JAMA Psychiatry 2016;73:388-95.

10. Hayley AC, Stough C, Downey LA. DSM-5 cannabis use disorder, substance use and DSM-5 specific substance-use disorders: Evaluating comorbidity in a population-based sample. Eur Neuropsychopharmacol 2017;27:732-43.

11. Whiting PF, Wolff RF, Deshpande S, et al. Cannabinoids for Medical Use: A Systematic Review and Meta-analysis. JAMA 2015;313:2456-73.
12. Hill KP. Medical Marijuana for Treatment of Chronic Pain and Other Medical and Psychiatric Problems: A Clinical Review. JAMA 2015;313:2474-83.

13. Karila L, Roux $P$, Rolland $B$, et al. Acute and long-term effects of cannabis use: a review. Curr Pharm Des 2014;20:4112-8.

14. Schreiner AM, Dunn ME. Residual effects of cannabis use on neurocognitive performance after prolonged abstinence: a metaanalysis. Exp Clin Psychopharmacol 2012;20:420-9.

15. Volkow ND. Effects of Cannabis Use on Human Behavior-Reply. JAMA Psychiatry 2016;73:996.

16. Lynskey MT, Glowinski AL, Todorov AA, et al. Major depressive disorder, suicidal ideation, and suicide attempt in twins discordant for cannabis dependence and early-onset cannabis use. Arch Gen Psychiatry 2004;61:1026-32.

17. Kim HM, Smith EG, Stano CM, et al. Validation of key behaviourally based mental health diagnoses in administrative data: suicide attempt, alcohol abuse, illicit drug abuse and tobacco use. BMC Health Serv Res 2012;12:18.

18. McCormick N, Lacaille D, Bhole V, et al. Validity of myocardial infarction diagnoses in administrative databases: a systematic review. PLoS One 2014:9:e92286.

19. Dewland TA, Glidden DV, Marcus GM. Healthcare utilization and clinical outcomes after catheter ablation of atrial flutter. PLoS One 2014;9:e100509.

20. Dukes JW, Dewland TA, Vittinghoff E, et al. Access to alcohol and heart disease among patients in hospital: observational cohort study using differences in alcohol sales laws. BMJ 2016;353:i2714.

21. Dewland TA, Olgin JE, Vittinghoff $E$, et al. Incident atrial fibrillation among Asians, Hispanics, blacks, and whites. Circulation 2013;128:2470-7.

22. Birkmeyer JD, Siewers AE, Finlayson EV, et al. Hospital volume and surgical mortality in the United States. N Engl J Med 2002;346:1128-37

23. Gialdini G, Nearing $\mathrm{K}$, Bhave $\mathrm{PD}$, et al. Perioperative atrial fibrillation and the long-term risk of ischemic stroke. JAMA 2014;312:616-22.

24. Whitman IR, Agarwal V, Nah G, et al. Alcohol Abuse and Cardiac Disease. J Am Coll Cardiol 2017;69:13-24.

25. Williams AR, Olfson M, Kim JH, et al. Older, Less Regulated Medical Marijuana Programs Have Much Greater Enrollment Rates Than Newer 'Medicalized' Programs. Health Aff 2016;35:480-8.

26. Williams AR, Santaella-Tenorio J, Mauro CM, et al. Loose regulation of medical marijuana programs associated with higher rates of adult marijuana use but not cannabis use disorder. Addiction 2017;112:1985-91. 